\title{
Quasiconvex envelopes of energies for nematic elastomers in the small strain regime and applications
}

\author{
Pierluigi Cesana* ${ }^{\dagger} \quad$ Antonio DeSimone ${ }^{\ddagger} \S$
}

September 10, 2010

Preprint SISSA no. 62/2010/M

\begin{abstract}
We provide some explicit formulas for the quasiconvex envelope of energy densities for nematic elastomers in the small strain regime and plane strain conditions. We then demonstrate their use as a powerful tool for the interpretation of mechanical experiments.
\end{abstract}

\section{Keywords}

Nematic elastomers; Hyperelasticity; Relaxation; Quasiconvexity.

PACS Physics and Astronomy Classification Scheme

61.30.-v Liquid crystals

61.30.Dk Continuum models and theories of liquid crystal structure 83.80.Va Elastomeric polymers

AMS Subject Classification Numbers

74-xx Mechanics of deformable solids

76A15 Liquid crystals

*SISSA, Trieste, Italy, cesana@sissa.it

${ }^{\dagger}$ Current address: BCAM, Bilbao, Spain

${ }^{\ddagger}$ SISSA, Trieste, Italy, desimone@sissa.it

${ }^{\S}$ Corresponding author 


\section{Contents}

1 Introduction: nematic elastomers 3

2 Energies and their quasiconvex envelopes 5

$\begin{array}{lll}3 & \text { Plane strain conditions } & 7\end{array}$

4 Application: discussion of some mechanical experiments 12

5 Appendix 1: Calculus of Variations tools 19

6 Appendix 2: relaxation theorems 22

6.1 Compressible elastomers . . . . . . . . . . . . . 24

6.2 Incompressible elastomers . . . . . . . . . . . . 25

6.3 Summary . . . . . . . . . . . . . 26 


\section{Introduction: nematic elastomers}

Nematic elastomers are polymeric materials with embedded nematic mesogens. Their mechanical response is governed by the coupling of rubber elasticity with the orientational order of a liquid crystalline system. Nematic elastomers exhibit large spontaneous deformations, which can be triggered and controlled by many different means (temperature, electric fields, irradiation by UV light). These properties make them interesting as materials for fast soft actuators and justify the considerable attention that they have attracted in recent years. The reader is referred to the monograph by Warner and Terentjev [24] for a thorough introduction to the physics of nematic elastomers, and for an extensive list of references.

While commercial applications exploiting their properties are still lacking, nematic elastomers are playing an important role as a model system for the study of the mechanics of phase transforming materials. In these materials, because of the symmetries of the phase transformation underlying their unusual mechanical properties, material instabilities are ubiquitous. Seen from the point of view of hyperelasticity, this means that the energy densities capable of reproducing the elastic response of phase transforming materials lack the convexity properties necessary to guarantee material stability. On the other hand, recent advances in the Calculus of Variations allow us to show that suitable convex envelopes of the energy densities (the so called quasiconvex envelopes) may provide valuable insight on the mechanical response of these materials. These techniques have been applied with considerable success in a variety of physical systems including nematic elastomers, shape memory alloys, magnetostrictive or ferreolectric materials, crystal plasticity (see [3] for a review). In this paper, we apply this approach to nematic elastomers in the small strain regime (geometrically linear theory), restricting our attention to plane strain conditions for simplicity.

The main accomplishments of this paper are the following. First, we obtain a new explicit formula for the quasiconvex envelope of an anisotropic energy density proposed to model nematic elastomers within the framework of geometrically linear theories. As observed already in [4] and [15], the use of anisotropic energies is crucial to avoid that imposed stretches may be accommodated at zero stress (ideally soft response), and to obtain results in agreement with the available experimental evidence (in particular, non vanishing shear moduli in the natural state of the material, see [21]). The use of quasiconvex envelopes to interpret mechanical tests is illustrated in Section 4. There, we discuss in detail the implications of our results for the understanding of experiments probing the mechanical response of nematic elastomers. Considerable insight is gained thanks to the availability of explicit formulas for the relaxed energy. In particular, we are able to give a simple characterization of stress-strain curves for specimens responding to the applied loads through deformations that are macroscopically homoge- 
noeus, but are spatially modulated at small length scales by systems of shear bands (usually called stripe domains in the nematic elastomer literature, see $[23,16])$.

In addition, we discuss the relationship between the energies and quasiconvex envelopes of the geometrically linear theory with their counterparts in the fully nonlinear theory. The availability of explicit formulas in both regimes is a rather unique feature of nematic elastomers, and a fortunate circumstance. We find that the insight deriving from the possibility of comparing them against each other, and with results from experimental evidence is particularly enlightening.

While the geometrically linear theory has obvious limitations (see, e.g., [3]), it is a very valuable conceptual tool in the study of phase transforming materials. It is simpler in many (though not all) respects, it is familiar to larger groups of users, the resulting energy landscape has an easier geometric structure, rigorous mathematical results available for it (some of which are first proved in this paper) are more complete. Most importantly, the linear theory lends itself more easily to the exploration of model extensions such as, for example, accounting for the effects of applied electric fields, including curvature elasticity terms typical of liquid crystals, modeling rate effects and viscous relaxation to equilibrium. All these are easily handled by simply adding new terms in the governing energy and by introducing appropriate dissipation potentials (see, e.g., [17]), while exploring deeply nonlinear regimes may require more complex coupling schemes. In all these extensions, the insight on energetically optimal states deriving from the explicit knowledge of the quasiconvex envelope of the elastic energy density proves to be a very valuable tool.

The rest of the paper is organized as follows. In Section 2 we recall some model energy densities for nematic elastomers, introduce the notion of quasiconvex envelope, and illustrate it by comparing two corresponding isotropic expressions arising from the geometrically linear and the fully nonlinear theory. In Section 3 we consider plane strain conditions, and discuss a new formula for the quasiconvex envelope of an anisotropic energy density. This formula is then applied in Section 4, where we simulate some of the experiments used to probe the mechanical properties of nematic elastomers.

The paper is written in the language of Continuum Mechanics. We have included also rigorous proofs of our results, which require some familiarity with advanced tools from the Calculus of Variations. This more mathematical part is contained in two Appendices, which can be skipped by readers wishing to concentrate only on the physical significance of our results and on their implications on the mechanical response of nematic elastomers. Parts of this material, such as the lamination construction contained in Proposition 4 of Section 6 , are however very useful to gain a deeper understanding of the material instabilities which make the mechanical response of nematic elastomers so interesting. 


\section{Energies and their quasiconvex envelopes}

Following [15], we are interested in three model expressions for the energy density of a nematic elastomer in the small strain regime (geometrically linear theory). The first one is

$$
\tilde{\Phi}(\mathbf{E}, \mathbf{n})=\mu\left|\mathbf{E}_{d}-\mathbf{E}_{0}(\mathbf{n})\right|^{2}+\frac{1}{2} \kappa(\operatorname{tr} \mathbf{E})^{2},
$$

where $\mathbf{E}_{d}=\mathbf{E}-\frac{1}{3} \operatorname{tr}(\mathbf{E}) \mathbf{I}$ is the deviatoric part of the infinitesimal strain $\mathbf{E}$,

$$
\mathbf{E}_{0}(\mathbf{n})=\frac{3}{2} \gamma\left(\mathbf{n} \otimes \mathbf{n}-\frac{1}{3} \mathbf{I}\right)
$$

is the (spontaneous or) stress-free strain associated with the orientation $\mathbf{n}$ of the nematic director (a unit vector field: we will emphasize this by writing $|\mathbf{n}|=1$ or, equivalently, $\left.\mathbf{n} \in \mathbb{S}^{2}\right)$. The positive scalars $\gamma, \mu, \kappa$ are material parameters controlling the magnitude of the spontaneous strain, the shear modulus, and the bulk modulus, respectively.

The second model expression is the incompressible version of (1), namely,

$$
\Phi(\mathbf{E}, \mathbf{n})=\mu\left|\mathbf{E}_{d}-\mathbf{E}_{0}(\mathbf{n})\right|^{2}, \quad \text { if } \operatorname{tr} \mathbf{E}=\operatorname{div} \mathbf{u}=0
$$

and $\Phi(\mathbf{E}, \mathbf{n})=+\infty$ if $\operatorname{tr} \mathbf{E} \neq 0$. Finally the third one is a correction of (3) obtained by adding an anisotropic term

$$
\Phi_{\beta}(\mathbf{E}, \mathbf{n})=\Phi(\mathbf{E}, \mathbf{n})+\beta \mu\left|\mathbf{E}_{d}-\mathbf{E}_{0}\left(\mathbf{e}_{2}\right)\right|^{2},
$$

where $\beta>0$ is a dimensionless parameter quantifying the strength of the anisotropic correction and $\mathbf{e}_{i}, i=1,2,3$ are the unit vectors of the canonical orthonormal basis.

The three energies above arise as small strain limits of three corresponding model expressions proposed by Warner, Terentjev, and coworkers to model nematic elastomers in the large deformation regime [5, 23, 24]. They can be justified either by Taylor expansion [15], or by Gamma-convergence arguments [2]. In what follows, we will be mostly concerned with (3) and (4), describing the incompressible case.

We now consider the smallest energy density achievable by a system governed by energy (3) if it is allowed to freely adjust $\mathbf{n}$, at fixed $\mathbf{E}$. Setting

$$
\varphi(\mathbf{E}):=\inf _{\mathbf{n} \in S^{2}} \Phi(\mathbf{E}, \mathbf{n}),
$$

it is easy to show that

$$
\varphi(\mathbf{E})=\mu\left[\left(e_{\max }-\gamma\right)^{2}+\left(e_{\operatorname{mid}}+\frac{1}{2} \gamma\right)^{2}+\left(e_{\min }+\frac{1}{2} \gamma\right)^{2}\right], \quad \text { if } \operatorname{tr} \mathbf{E}=0
$$


and $\varphi(\mathbf{E})=+\infty$, if $\operatorname{tr} \mathbf{E} \neq 0$. Here $e_{\max } \geq e_{\text {mid }} \geq e_{\min }$ are the ordered eigenvalues of $\mathbf{E}$ (principal strains). Using that $e_{\max }+e_{\operatorname{mid}}+e_{\min }=0$ for strains with finite energy, we can rewrite (6) in the following way

$$
\varphi(\mathbf{E})=\frac{3}{2} \mu\left(e_{\min }+\frac{1}{2} \gamma\right)^{2}+\frac{1}{2} \mu\left(e_{\min }+2 e_{\max }-\frac{3}{2} \gamma\right)^{2}, \quad \text { if } \operatorname{tr} \mathbf{E}=0
$$

Next, we seek the smallest energy density achievable by the system if it is allowed to develop microstructure (i.e., displacements that are spatially modulated at small length scales) at fixed average strain $\mathbf{E}$. This is given by $\varphi^{\mathrm{qc}}(\mathbf{E})$, namely, the quasiconvex envelope $\varphi^{\mathrm{qc}}$ of $\varphi$ evaluated at $\mathbf{E}$. In formulas,

$$
\varphi^{\mathrm{qc}}(\mathbf{E}):=\inf _{\mathbf{w}} \frac{1}{|\omega|} \int_{\omega} \varphi(\mathbf{E}+\nabla \mathbf{w}(x)) d x,
$$

where $\omega \subset \mathbb{R}^{3}$ is an arbitrary (Lipschitz) domain (it can be shown that (8) does not depend on shape and size of the test region $\omega),|\omega|$ is its volume, and $\mathbf{w}$ is an arbitrary perturbation (a Lipschitz-continuous displacement field perturbing the affine state with strain $\mathbf{E}$ ) vanishing on $\partial \omega$ and such that $\operatorname{div} \mathbf{w}=\operatorname{tr} \nabla \mathbf{w}=0$. In writing (8), and throughout the paper, the trivial extension of $\varphi$ to non-symmetric matrices, defined by $\varphi_{\mathrm{ex}}(\mathbf{A})=\varphi(\operatorname{sym} \mathbf{A})$ for every matrix $\mathbf{A}$, where $\operatorname{sym} \mathbf{A}=\left(\mathbf{A}+\mathbf{A}^{T}\right) / 2$, is denoted simply by $\varphi$.

Stable materials are characterized by $\varphi^{\mathrm{qc}} \equiv \varphi$. If, for some $\mathbf{E}, \varphi^{\mathrm{qc}}(\mathbf{E})<$ $\varphi(\mathbf{E})$, then the state of homogeneous deformation $\mathbf{E}$ is unstable: the material shows an energetic preference to develop spatially modulated deformations (typically, shear bands) at fixed average deformation $\mathbf{E}$. This is the case for nematic elastomers.

An explicit formula for the quasiconvex envelope of $\varphi$ has been obtained in [7], see Section 5. According to this formula, $\varphi^{\mathrm{qc}}(\mathbf{E})=+\infty$, if $\operatorname{tr} \mathbf{E} \neq 0$ while, if $\operatorname{tr} \mathbf{E}=0$, we have

$$
\varphi^{\mathrm{qc}}(\mathbf{E})= \begin{cases}0 & \text { if }-e_{\min } \leq \frac{\gamma}{2}, \\ \varphi(\mathbf{E}) & \text { if } e_{\max } \geq-\frac{1}{2} e_{\min }+\frac{3}{4} \gamma \\ \frac{3}{2} \mu\left(e_{\min }+\frac{1}{2} \gamma\right)^{2} & \text { else. }\end{cases}
$$

Formula (9) above provides the small strain counterpart of the relaxation result in [14]. The most basic formula for the analysis of the large deformation regime is the isotropic energy density

$$
W(\mathbf{F})=\frac{1}{2} \mu a^{1 / 3}\left(\lambda_{\text {min }}^{2}+\lambda_{\text {mid }}^{2}+\frac{1}{a} \lambda_{\max }^{2}-3 a^{-1 / 3}\right), \quad \text { if } \operatorname{det} \mathbf{F}=1
$$

and $W(\mathbf{F})=+\infty$, if $\operatorname{det} \mathbf{F} \neq 1$. Here $\mathbf{F}$ is the deformation gradient, $\lambda_{\max } \geq$ $\lambda_{\text {mid }} \geq \lambda_{\text {min }}$ are the ordered singular values of $\mathbf{F}$ (principal stretches), and $a>1$ a material parameter controlling the magnitude of the spontaneous stretches. This is obtained from the celebrated trace formula proposed in [5] 
by a change of reference configuration and by minimizing over the nematic degrees of freedom, as first shown in [12]. An equivalent expression for (10) is

$$
W(\mathbf{F})=\frac{1}{2} \mu \operatorname{tr}\left(\mathbf{B B}_{0}^{-1}(\mathbf{n})\right), \quad \text { if } \operatorname{det} \mathbf{F}=1
$$

where $\mathbf{B}=\mathbf{F F}^{T}$,

$$
\mathbf{B}_{0}(\mathbf{n})=\left(\mathbf{V}_{0}(\mathbf{n})\right)^{2}=\left(a^{1 / 3} \mathbf{n} \otimes \mathbf{n}+a^{-1 / 6}(\mathbf{I}-\mathbf{n} \otimes \mathbf{n})\right)^{2},
$$

and $\mathbf{I}$ is the identity, see $[15,13]$. The instructive comparison between (3) and (11) shows that they both express an energetic penalty for deformations relative to a preferred one associated with $\mathbf{n}$. This relative deformation measure is obtained through the composition with an inverse in the nonlinear theory, through a difference in the case of linear kinematics. The quasiconvex envelope of $W$ is $W^{\mathrm{qc}}(\mathbf{F})=+\infty$, if $\operatorname{det} \mathbf{F} \neq 1$ while, for $\operatorname{det} \mathbf{F}=1$ it is given by the formula

$$
W^{\mathrm{qc}}(\mathbf{F})= \begin{cases}0 & \text { if } 1 / \lambda_{\min } \leq a^{1 / 6} \\ W(\mathbf{F}) & \text { if } \lambda_{\max } \geq a^{1 / 4}\left(\frac{1}{\lambda_{\min }}\right)^{1 / 2} \\ \frac{1}{2} \mu a^{1 / 3}\left(\frac{2}{a^{1 / 2} \lambda_{\min }}+\lambda_{\min }^{2}-3 a^{-1 / 3}\right) & \text { else. }\end{cases}
$$

The energies described above are plotted in figures 1 and 2, together with their quasiconvex envelopes. The only zero of $W$ in the $\left(\lambda_{\max }, \lambda_{\min }^{-1}\right)$-plane is $\left(a^{1 / 3}, a^{1 / 6}\right)$. The only zero of $\varphi$ in the $\left(e_{\max },-e_{\min }\right)$-plane is $(\gamma, \gamma / 2)$. The graphs show that the geometrically linear theory provides a faithful representation of the energy landscape, provided that all the deformations involved are small. This requires, in particular, that $a^{1 / 3}=1+\gamma, \gamma<<1$.

\section{Plane strain conditions}

We consider now the case of plane strain, with the director moving in the plane of strain. In formulas,

$$
\begin{gathered}
\mathbf{n} \in \mathbb{S}^{1}=\left\{\mathbf{n} \in \operatorname{span}\left\{\mathbf{e}_{1}, \mathbf{e}_{2}\right\},|\mathbf{n}|=1\right\} \\
E_{33}=-\frac{\gamma}{2}, \quad E_{13}=E_{23}=0 \\
\operatorname{tr} \mathbf{E}=0
\end{gathered}
$$

We can then parametrize $\mathbf{E}$ as follows

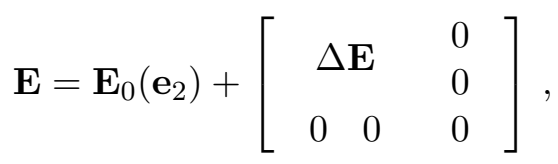




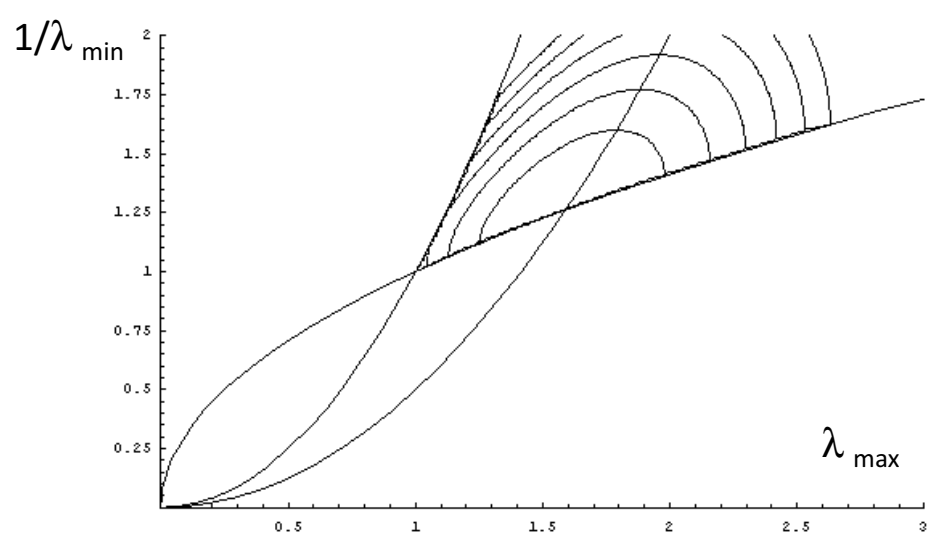

$1 / \lambda_{\min }$

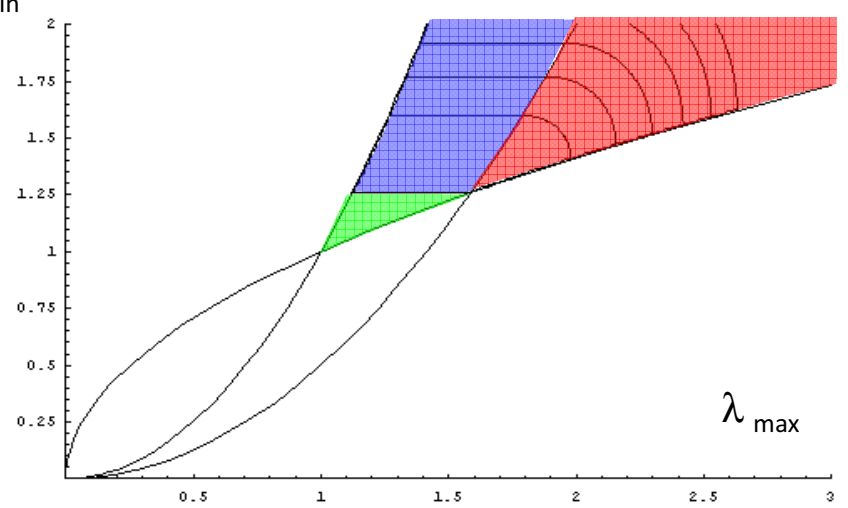

Figure 1: Level curves of the energy density (10) and of its quasiconvex envelope (13) (fully nonlinear theory). 

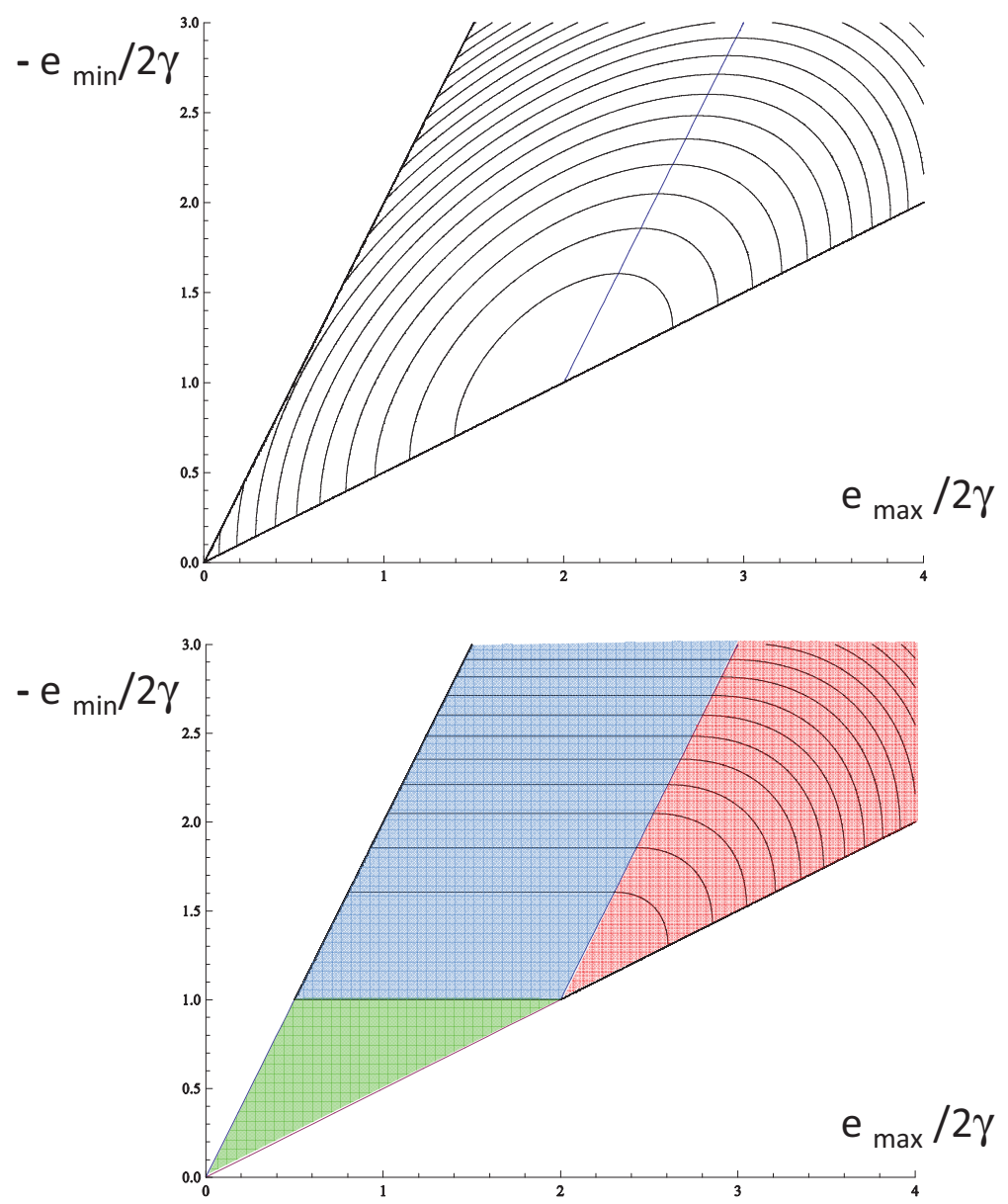

Figure 2: Level curves of the energy density (7) and of its quasiconvex envelope (9) (geometrically linear theory). 
where $\Delta \mathbf{E}$ is a $2 \times 2$ symmetric and traceless matrix. More explicitly,

$$
\mathbf{E}=\mathbf{E}_{0}\left(\mathbf{e}_{2}\right)+\frac{3}{2} \gamma\left[\begin{array}{ccc}
\varepsilon & \delta & 0 \\
\delta & -\varepsilon & 0 \\
0 & 0 & 0
\end{array}\right]=\frac{3}{2} \gamma\left[\begin{array}{ccc}
\varepsilon-1 / 3 & \delta & 0 \\
\delta & 2 / 3-\varepsilon & 0 \\
0 & 0 & -1 / 3
\end{array}\right]
$$

notice that, for $\delta=0, \mathbf{E}$ goes from $\mathbf{E}_{0}\left(\mathbf{e}_{2}\right)$ to $\mathbf{E}_{0}\left(\mathbf{e}_{1}\right)$ as $\varepsilon$ spans the interval from 0 to 1.

In the plane strain conditions described by (15)-(16) one has that either $e_{\min }=-\gamma / 2, e_{\operatorname{mid}}=\gamma / 2-e_{\max }$ (this is when $\left.e_{\max } \leq \gamma\right)$ or $e_{\operatorname{mid}}=-\gamma / 2$, $e_{\min }=\gamma / 2-e_{\max }$ (this is when $e_{\max } \geq \gamma$ ). We therefore have

$$
\varphi(\mathbf{E})=\inf _{\mathbf{n} \in \mathbb{S}^{1}} \Phi(\mathbf{E}, \mathbf{n})=2 \mu\left(e_{\max }-\gamma\right)^{2}, \quad \text { if } \operatorname{tr} \mathbf{E}=0
$$

and $\varphi(\mathbf{E})=+\infty$, if $\operatorname{tr} \mathbf{E} \neq 0$. Moreover, we have

$$
\varphi_{\beta}(\mathbf{E})=\inf _{\mathbf{n} \in \mathbb{S}^{1}} \Phi_{\beta}(\mathbf{E}, \mathbf{n})=\varphi(\mathbf{E})+\beta \mu\left|\mathbf{E}-\mathbf{E}_{0}\left(\mathbf{e}_{2}\right)\right|^{2}, \quad \text { if } \operatorname{tr} \mathbf{E}=0
$$

and $\varphi_{\beta}(\mathbf{E})=+\infty$, if $\operatorname{tr} \mathbf{E} \neq 0$.

It is useful to write the energies above as functions of the parameters $\varepsilon$ and $\delta$ appearing in (18). We observe that

$$
e_{\max }(\mathbf{E}(\varepsilon, \delta))=\frac{3}{2} \gamma\left(\frac{1}{6}+r(\varepsilon, \delta)\right)
$$

where

$$
r(\varepsilon, \delta):=\sqrt{\left(\varepsilon-\frac{1}{2}\right)^{2}+\delta^{2}}
$$

is the distance of point $(\varepsilon, \delta)$ from $(1 / 2,0)$, and write

$$
\begin{gathered}
f(\varepsilon, \delta)=\varphi(\mathbf{E}(\varepsilon, \delta))=\frac{9}{2} \mu \gamma^{2}\left(r(\varepsilon, \delta)-\frac{1}{2}\right)^{2}, \\
f_{\beta}(\varepsilon, \delta)=\varphi_{\beta}(\mathbf{E}(\varepsilon, \delta))=f(\varepsilon, \delta)+\frac{9}{2} \beta \mu \gamma^{2}\left(\varepsilon^{2}+\delta^{2}\right) .
\end{gathered}
$$

We can compute explicit formulas for the quasiconvex envelope of these two energy densities. The relaxation theorem proved in Section 6 shows that $\varphi_{\beta}^{q c}(\mathbf{E})=+\infty$, if $\operatorname{tr} \mathbf{E} \neq 0$, while if $\operatorname{tr} \mathbf{E}=0$, then

$$
\varphi_{\beta}^{q c}(\mathbf{E})= \begin{cases}\varphi_{\beta}(\mathbf{E}) & \text { if } e_{\max } \geq \frac{1}{4}\left(1+\frac{3}{1+\beta}\right) \gamma \\ \frac{9}{2} \beta \mu \gamma^{2}\left(\frac{E_{11}-E_{22}}{3 \gamma}+\frac{1}{2}-\frac{1}{4} \frac{\beta}{1+\beta}\right) & \text { else. }\end{cases}
$$

Equivalently,

$\varphi_{\beta}^{q c}(\mathbf{E}(\varepsilon, \delta))=f_{\beta}^{c}(\varepsilon, \delta)= \begin{cases}f_{\beta}(\varepsilon, \delta) & \text { if }\left(\left(\varepsilon-\frac{1}{2}\right)^{2}+\delta^{2}\right)^{1 / 2} \geq \frac{1}{2} \frac{1}{1+\beta} \\ \frac{9}{2} \beta \mu \gamma^{2}\left(\varepsilon-\frac{1}{4} \frac{\beta}{1+\beta}\right) & \text { else }\end{cases}$ 

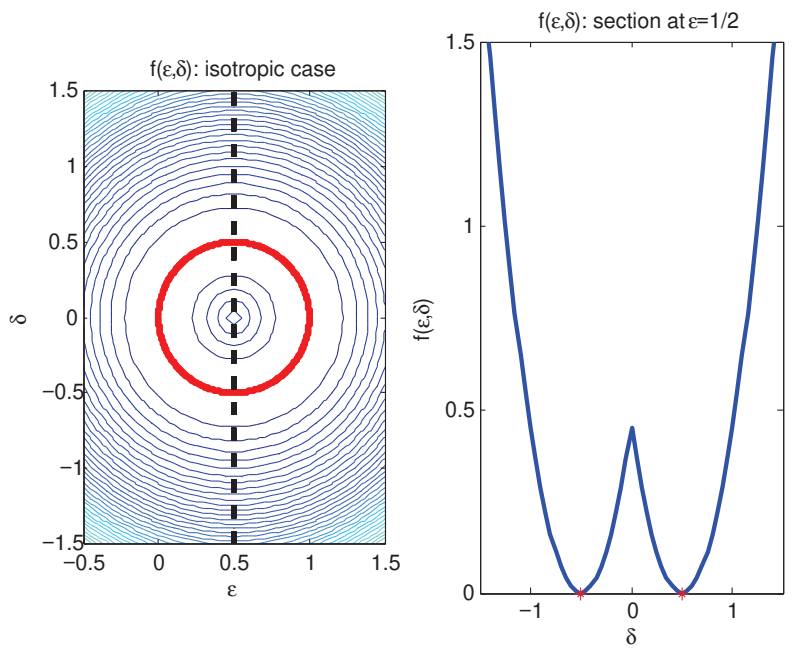

Figure 3: Level curves of the isotropic energy density $\varphi$ in $(\varepsilon, \delta)$ plane, and section at $\varepsilon=1 / 2$.

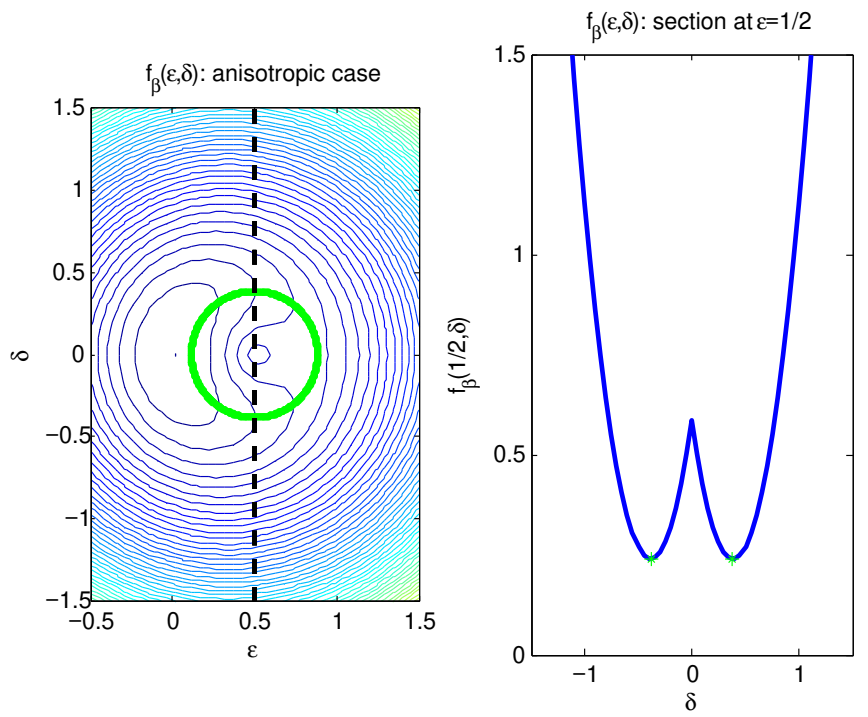

Figure 4: Level curves of the anisotropic energy density $\varphi_{\beta}$ in $(\varepsilon, \delta)$ plane, and section at $\varepsilon=1 / 2$. 

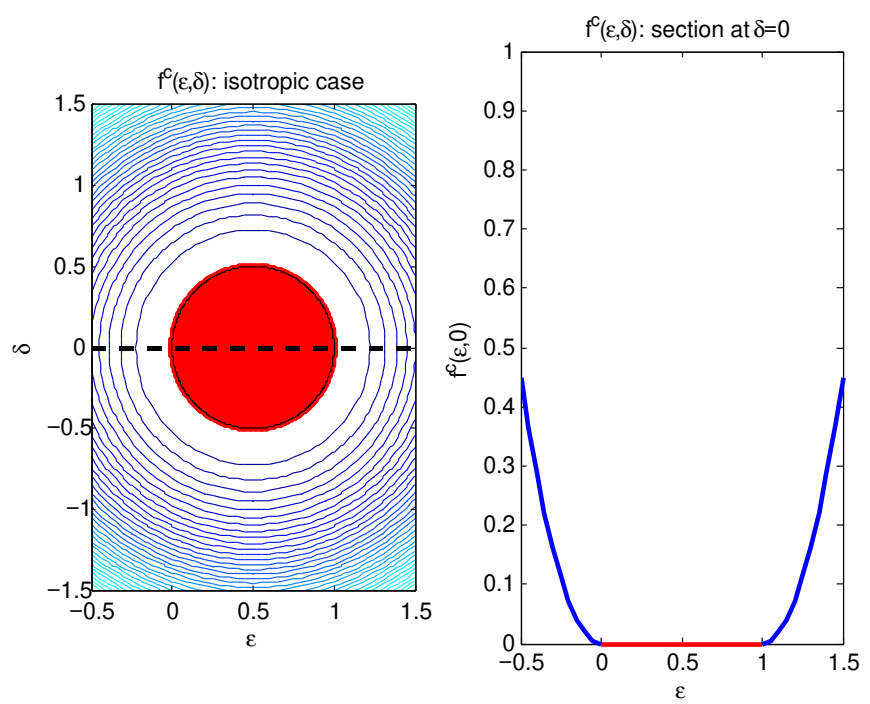

Figure 5: Level curves of the quasiconvex envelope of the isotropic energy density $\varphi$ in $(\varepsilon, \delta)$ plane, and section at $\delta=0$.

where $f_{\beta}^{c}$ is the convex envelope of $f_{\beta}$.

The isotropic case is obtained either from the result for the anisotropic case, by setting $\beta=0$, or by inserting the plane strain conditions (15)-(16) in the general relaxation result (9). We thus have that, for $\operatorname{tr} \mathbf{E}=0$,

$$
\varphi^{q c}(\mathbf{E})= \begin{cases}2 \mu\left(e_{\max }-\gamma\right)^{2} & \text { if } e_{\max } \geq \gamma \\ 0 & \text { else. }\end{cases}
$$

Equivalently,

$$
\varphi^{q c}(\mathbf{E}(\varepsilon, \delta))=f^{c}(\varepsilon, \delta)= \begin{cases}f(\varepsilon, \delta) & \text { if }\left(\left(\varepsilon-\frac{1}{2}\right)^{2}+\delta^{2}\right)^{1 / 2} \geq \frac{1}{2} \\ 0 & \text { else }\end{cases}
$$

where $f_{\beta}^{c}$ is the convex envelope of $f_{\beta}$.

Level set plots and sections of the energy landscape associated with the energy densities introduced above are shown in figures $3-6$. They provide a deep and compact view of the behavior of a material governed by energies (19) and (20), as shown in the next Section.

\section{Application: discussion of some mechanical ex- periments}

We apply the results of the previous sections to study the behavior of a specimen of size $L \times 1$ tested in plane strain conditions. We start with a 

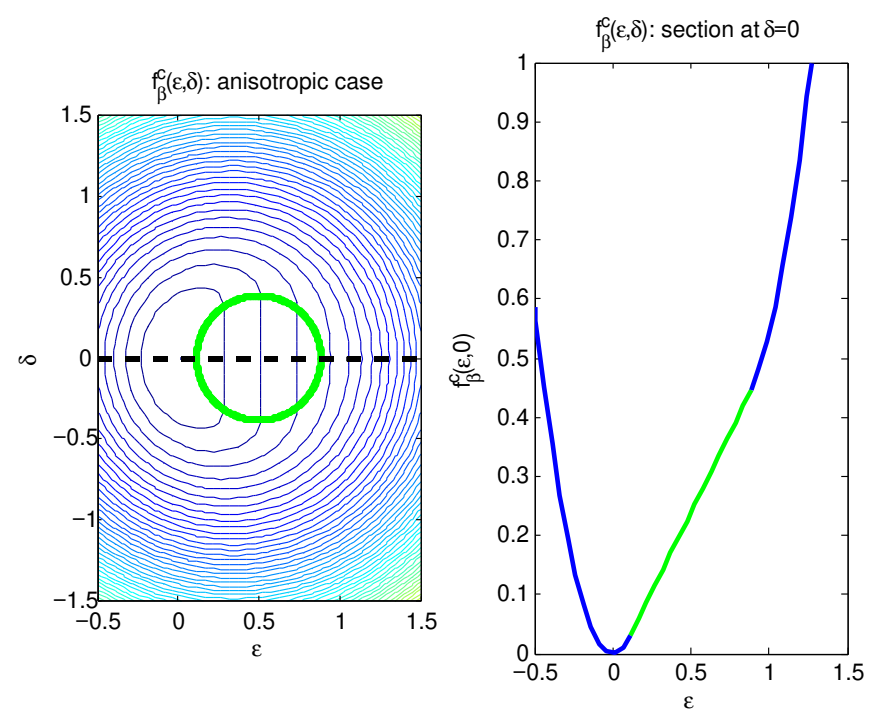

Figure 6: Level curves of the quasiconvex envelope of the anisotropic energy density $\varphi_{\beta}$ in $(\varepsilon, \delta)$ plane, and section at $\delta=0$.

(laterally) unconstrained extension along $\mathbf{e}_{1}$ : a pure shear in the language of Treloar (notice that a uniaxial extension along $\mathbf{e}_{1}$ requires no confinement also along $x_{3}$; since this may be incompatible with plane strain conditions we will not deal with this case here, and refer the reader to $[9,10]$ and $[4]$ for results on uniaxial extension in thin film geometries).

Using as reference configuration the stress-free strain corresponding to $\mathbf{n} \equiv \mathbf{e}_{2}$ (i.e., $\varepsilon=\delta=0$ ), we impose displacements $u_{1}\left(0, x_{2}\right) \equiv 0, u_{1}\left(L, x_{2}\right) \equiv$ $u$ on the vertical edges and leave the horizontal edges free (see Figure 4). We look for homogeneously deformed states, with

$$
\varepsilon=\varepsilon(u)=\frac{2}{3 \gamma} \frac{u}{L}
$$

and $\delta$ a constant to be determined. We use in all our calculations the anisotropic expressions $f_{\beta}$ and $f_{\beta}^{c}$. The results for the isotropic case are easily obtained by setting $\beta=0$.

The energy (per unit thickness in the $x_{3}$ direction) stored in the specimen is $L \times f_{\beta}(\varepsilon(u), \delta)$ and it is made stationary by equilibrium states. Solving for $\delta_{\text {eq }}$ in

$$
\left.\frac{\partial}{\partial \delta} f_{\beta}(\varepsilon(u), \delta)\right|_{\delta=\delta_{\mathrm{eq}}}=0
$$

we obtain $\delta_{\text {eq }}=0$ (this is a possible equilibrium for any value of $\varepsilon$ ) and, 


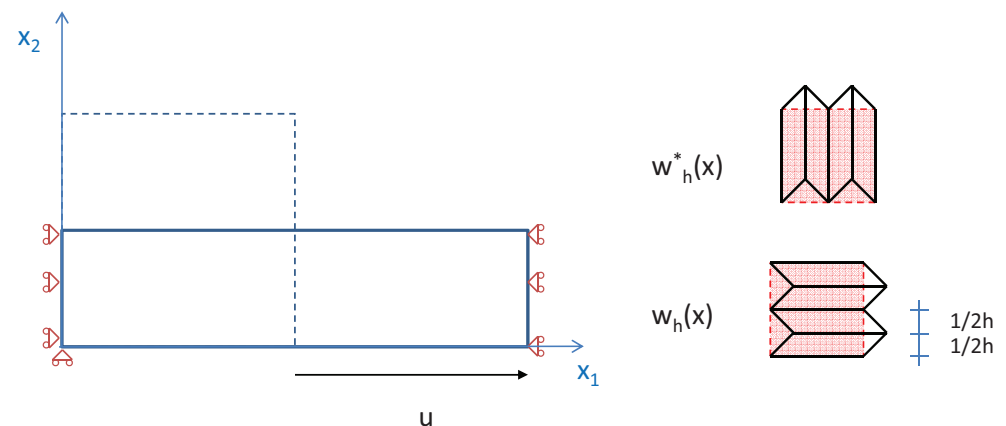

Figure 7: Geometry of plane-strain extension experiments. On the right, geometry of localized shear band patterns.

provided that

$$
\varepsilon_{\beta}^{-}:=\frac{1}{2} \frac{\beta}{1+\beta}<\varepsilon<\frac{1}{2} \frac{2+\beta}{1+\beta}=: \varepsilon_{\beta}^{+}
$$

we have in addition sheared equilibrium states with

$$
\delta_{\mathrm{eq}}= \pm \bar{\delta}= \pm \sqrt{\left(\frac{1}{2} \frac{1}{1+\beta}\right)^{2}-\left(\varepsilon-\frac{1}{2}\right)^{2}}, \quad \varepsilon_{\beta}^{-}<\varepsilon<\varepsilon_{\beta}^{+} .
$$

Since

$$
\left.\frac{\partial^{2}}{\partial \delta^{2}} f_{\beta}(\varepsilon(u), \delta)\right|_{\delta=0}=9 \mu \gamma^{2}\left(1+\beta-\frac{1}{2} \frac{1}{|\varepsilon-1 / 2|}\right)<0 \text { for } \varepsilon_{\beta}^{-}<\varepsilon<\varepsilon_{\beta}^{+},
$$

unsheared homogeneous states are unstable in the range $\varepsilon_{\beta}^{-}<\varepsilon(u)<\varepsilon_{\beta}^{+}$. By contrast, when available, sheared homogeneous states are always stable. These results are easily checked by using the graphs of $f_{\beta}$ and $f$ shown in figures 5 and 6 .

If we provisionally assume that suitable constraints can keep the system in the unstable unsheared equilibria, we can use conservation of energy to evaluate the force per unit area, $\sigma(u)$, exerted along $\mathbf{e}_{1}$ on the vertical edges. From

$$
\sigma(u)=\frac{d}{d u}\left(L f_{\beta}(\varepsilon(u), 0)\right)
$$




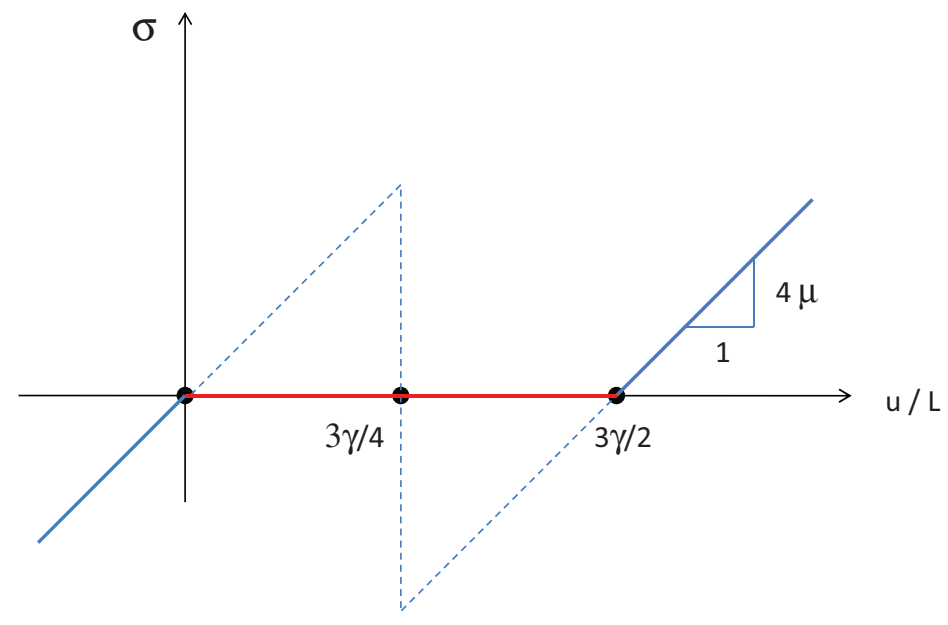

Figure 8: Stress-strain response in plane strain extension: isotropic case.

we obtain

$$
\sigma(u)=\left\{\begin{array}{cc}
4 \mu(1+\beta) \frac{u}{L} & \text { for } \frac{u}{L}<\frac{3}{4} \gamma \\
4 \mu(1+\beta) \frac{u}{L}-6 \mu \gamma & \text { for } \frac{u}{L}>\frac{3}{4} \gamma .
\end{array}\right.
$$

The plot of $\sigma$ against $u / L$ provides the stress-strain response of the (unsheared) system in plane-strain extension, and it is shown in figures 8 and 9 (with dashed lines in the unstable regions). We now explore the impact of material instabilities on the response of the system.

The availability of an explicit formula for the quasiconvex envelope of the energy density enables us to explore another class of (macroscopically) homogeneous and unsheared states, in which spatially modulated perturbations are allowed at a macroscopic scale. Let $\mathbf{u}$ be the affine displacement describing an unsheared state in the unstable region

$$
\mathbf{u}(x)=\mathbf{F} \mathbf{x}, \quad \mathbf{F}=\frac{3}{2} \gamma\left[\begin{array}{cc}
\varepsilon & 0 \\
0 & -\varepsilon
\end{array}\right], \quad \varepsilon_{\beta}^{-}<\varepsilon<\varepsilon_{\beta}^{+} .
$$

We perform a lamination construction to find a perturbation displacement $\mathbf{w}_{h}$, modulated at a length scale of order $1 / h$, which lowers the energy of the system while keeping the (macroscopic) average displacement gradient equal to F. Using the formulas from Proposition 4 (with the caveat that, since we are now using as reference configuration the one with $\varepsilon=\delta=0$, 


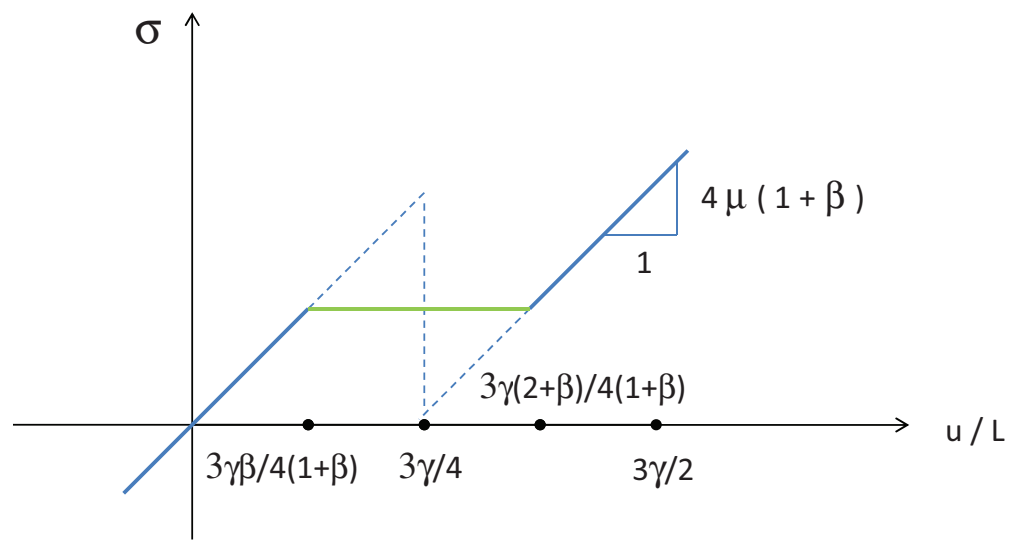

Figure 9: Stress-strain response in plane strain extension: anisotropic case.

$\mathbf{E}_{0}\left(\mathbf{e}_{2}\right)$ drops out from all formulas), we set

$$
\Delta \mathbf{E}_{1}=\frac{3}{2} \gamma\left[\begin{array}{cc}
\varepsilon & \bar{\delta} \\
\bar{\delta} & -\varepsilon
\end{array}\right], \Delta \mathbf{E}_{2}=\frac{3}{2} \gamma\left[\begin{array}{cc}
\varepsilon & -\bar{\delta} \\
-\bar{\delta} & -\varepsilon
\end{array}\right], \Delta \mathbf{W}=\frac{3}{2} \gamma\left[\begin{array}{cc}
0 & \bar{\delta} \\
-\bar{\delta} & 0
\end{array}\right],
$$

where $\bar{\delta}$ is given in (31). Moreover, we set

$$
\begin{gathered}
\mathbf{F}_{1}=\Delta \mathbf{E}_{1}+\Delta \mathbf{W}=\frac{3}{2} \gamma\left[\begin{array}{cc}
\varepsilon & 2 \bar{\delta} \\
0 & -\varepsilon
\end{array}\right]=\mathbf{F}+3 \gamma \bar{\delta} \mathbf{e}_{1} \otimes \mathbf{e}_{2}, \\
\mathbf{F}_{2}=\Delta \mathbf{E}_{2}-\Delta \mathbf{W}=\frac{3}{2} \gamma\left[\begin{array}{cc}
\varepsilon & -2 \bar{\delta} \\
0 & -\varepsilon
\end{array}\right]=\mathbf{F}-3 \gamma \bar{\delta} \mathbf{e}_{1} \otimes \mathbf{e}_{2} .
\end{gathered}
$$

Since $\mathbf{F}_{2}-\mathbf{F}_{1}=3 \gamma \bar{\delta} \mathbf{e}_{1} \otimes \mathbf{e}_{2}$ is a rank-one matrix, Hadamard's kinematic compatibility condition is satisfied and we can construct a continuous displacement $\mathbf{u}+\mathbf{w}_{h}$ whose gradient takes only the values $\mathbf{F}_{1}$ and $\mathbf{F}_{2}$. Indeed, setting

$$
\mathbf{w}_{h}(\mathbf{x})=\frac{1}{h} 3 \gamma \bar{\delta} \mathbf{e}_{1} \int_{0}^{h \mathbf{x} \cdot \mathbf{e}_{2}} \chi(s) d s,
$$

where $\chi$ is the one-periodic function such that

$$
\chi(s)=\left\{\begin{array}{cc}
1 & \text { if } s \in[0,1 / 2] \\
-1 & \text { if } s \in(1 / 2,1)
\end{array}\right.
$$

we have

$$
\nabla \mathbf{w}_{h}(\mathbf{x})=3 \gamma \bar{\delta} \mathbf{e}_{1} \otimes \chi\left(h \mathbf{x} \cdot \mathbf{e}_{2}\right) \mathbf{e}_{2}
$$


and, in turn,

$\nabla\left(\mathbf{u}+\mathbf{w}_{h}\right)=\left\{\begin{array}{l}\mathbf{F}_{1}=\mathbf{F}+3 \gamma \bar{\delta} \mathbf{e}_{1} \otimes \mathbf{e}_{2} \quad \text { in layers where } x_{2} \bmod h \in[0,1 / 2] \\ \mathbf{F}_{2}=\mathbf{F}-3 \gamma \bar{\delta} \mathbf{e}_{1} \otimes \mathbf{e}_{2} \quad \text { in layers where } x_{2} \bmod h \in(1 / 2,1) .\end{array}\right.$

This formula shows that $\mathbf{u}+\mathbf{w}_{h}$ has constant gradient in bands perpendicular to $\mathbf{e}_{2}$ of thickness $1 / 2 h$, with alternating values $\mathbf{F}_{1}$ and $\mathbf{F}_{2}$. Moreover, (40) shows that $\nabla \mathbf{w}_{h}$ takes opposite values $\pm 3 \gamma \bar{\delta} \mathbf{e}_{1} \otimes \mathbf{e}_{2}$ on bands of equal thickness, so that $\mathbf{w}_{u}$ converges uniformly to zero as $h$ tends to infinity, see Figure 4. As a consequence, $\mathbf{u}+\mathbf{w}_{h} \rightarrow \mathbf{u}$ uniformly in the limit $h \rightarrow \infty$.

Moving now to energetics, observe that

$$
\varphi_{\beta}\left(\mathbf{F}_{1}\right)=\varphi_{\beta}\left(\mathbf{F}_{2}\right)=\varphi_{\beta}^{q c}(\mathbf{F})=f_{\beta}^{c}(\varepsilon, 0) .
$$

This is clear from the construction of $\varphi_{\beta}^{q c}$, but also from the graph of $f_{\beta}^{c}$ in figure 6. Since $\nabla\left(\mathbf{u}+\mathbf{w}_{h}\right)$ takes only the values $\mathbf{F}_{1}$ and $\mathbf{F}_{2}$, the energy associated with $\mathbf{u}+\mathbf{w}_{h}$ is $L f_{\beta}^{c}(\varepsilon(u), 0)$, independent of $h$. We can thus attribute the same energy to the macroscopically homogeneous unsheared state obtained in the limit $h \rightarrow \infty$. Proceeding as in (33), the resulting stress-strain curve is obtained from

$$
\sigma(u)=\frac{d}{d u}\left(L f_{\beta}^{c}(\varepsilon(u), 0)\right)
$$

and plotted in figures 8 and 9 (with continuous lines). The curve in figure 9 shows the typical behavior observed in stretching experiments of nematic elastomers, with a plateau reached after a finite stress threshold [24]. Figure 8 represents an ideally soft limit, in which the stress threshold has disappeared. The curves resulting from (41) can be obtained from those resulting from (33) through Maxwell's equal area construction. As it is well known, this is precisely the result of passing from $f_{\beta}$ in equation (33) to its convex envelope $f_{\beta}^{c}$ in (42).

The fine scale modulations $\mathbf{w}_{h}$ represent a system of elastic shear bands, parallel to the direction of $\mathbf{e}_{1}$, and have been observed in stretching experiments on nematic elastomers. This is the stripe-domain instability, first reported in [18] and analyzed in [23]. As shown above, the homogeneously deformed, unsheared state is unstable towards formation of both global shears and finely modulated shear bands. When a system of such fine scale shear bands develops throughout the specimen, this can be (macroscopically) unsheared, and its stress-strain response would be the one shown in figures 8 and 9. It is important to recognize that there are other energetically optimal microstructures, different from $\mathbf{w}_{h}$, but they lead to the same macroscopic response at the level of stress-strain curves. For example, by replacing $\Delta \mathbf{W}$ in (36) with

$$
\Delta \mathbf{W}^{*}=\frac{3}{2} \gamma\left[\begin{array}{cc}
0 & -\bar{\delta} \\
\bar{\delta} & 0
\end{array}\right]
$$


and setting

$$
\mathbf{w}_{h}^{*}(\mathbf{x})=\frac{1}{h} 3 \gamma \bar{\delta} \mathbf{e}_{2} \int_{0}^{h \mathbf{x} \cdot \mathbf{e}_{1}} \chi(s) d s,
$$

we can construct a perturbation displacement in the form of vertical shear bands, as shown in Figure 4 . The field $\mathbf{u}+\mathbf{w}_{h}^{*}$ resolves the macroscopic gradient $\mathbf{F}$ using displacement gradients

$$
\mathbf{F}_{1,2}^{*}=\mathbf{F} \pm 3 \gamma \bar{\delta} \mathbf{e}_{2} \otimes \mathbf{e}_{1}
$$

and it has exactly the same energy as $\mathbf{u}+\mathbf{w}_{h}$. By multiplying $\mathbf{w}_{h}$ and $\mathbf{w}_{h}^{*}$ with suitable cut-off functions one may create local patches with shear bands oriented either horizontally or vertically, and even combine them to generate complex textures. For all these complex geometries, the resulting stress-strain response is still the one described by (42) and shown in figures 8 and 9.

Another important class of plane strain mechanical experiment is simple shear. We consider a shearing experiment in which a specimen, which has been previously stretched along $\mathbf{e}_{2}$ and is macroscopically unsheared as in Figure 4, experiences an additional imposed displacement given by $v_{1} \equiv 0$, $v_{2}\left(x_{1}, x_{2}\right)=x_{1} v / L$ at $x_{1}=0, L$, with $v / L$ small. The analysis of the case of simple shear with $v_{2} \equiv 0$ and $v_{1}\left(x_{1}, x_{2}\right)=x_{2} v$ at $x_{2}=0,1$ is analogous. Thanks to the availability of the explicit formula for $\varphi_{\beta}^{q c}$, we can estimate the shear modulus also for stretched states which are not homogeneous at the microscopic scale because of shear band texture. The relevant parameters in this experiment are $\delta=\delta(v)=(1 / 3 \gamma)(v / L)$ and $\varepsilon=\varepsilon(u)$. The shear modulus is

$$
G_{\beta}(\varepsilon)=\left.\frac{1}{9 \gamma^{2}} \frac{\partial^{2}}{\partial \delta^{2}} f_{\beta}^{c}(\varepsilon(u), \delta)\right|_{\delta=0}
$$

and more explicitly, using (32),

$$
G_{\beta}(\varepsilon)=\left\{\begin{array}{cc}
0 & \text { if } \varepsilon \in\left[\varepsilon_{\beta}^{-}, \varepsilon_{\beta}^{+}\right] \\
\mu\left(1+\beta-\frac{1}{2} \frac{1}{|\varepsilon-1 / 2|}\right)>0 & \text { otherwise }
\end{array}\right.
$$

with initial value

$$
G_{\beta}^{\|}:=G_{\beta}(0)=\mu \beta .
$$

The last two equations show that stretching a specimen starting from $\varepsilon=0$ causes the shear modulus to decrease from its initial value (44) and drop to zero at $\varepsilon=\varepsilon_{\beta}^{-}$, when the plateau in the stress-strain response is reached. This has been observed experimentally in [20]. It is instructive to compare the initial modulus (44) with the shear modulus $G_{\beta}^{\perp}$ for shears in the plane 13, perpendicular to the preferred orientation $\mathbf{e}_{2}$ of the nematic director. This is easily obtained from (4) as

$$
G_{\beta}^{\perp}=\mu(1+\beta)
$$


so that the ratio between the two moduli is

$$
\frac{G_{\beta}^{\|}}{G_{\beta}^{\perp}}=\frac{\beta}{1+\beta} .
$$

Values for this ratio around 0.5 have been measured experimentally in [21], and this requires values for $\beta$ of order one.

More complex experiments are of course possible, in which non homogeneous states of deformation are induced. A key example is stretching experiments in which clamps do not allow for lateral contraction. These type of experiments are harder to interpret (and less suitable to the evaluation of material parameters than the ones inducing homogeneous states of deformation), but provide stringent tests for the validity of a model. Numerical simulations taking correctly into account the energetics of systems of energetically optimal microscopic shear bands, without resolving them explicitly, can be set up by solving numerically the boundary value problem simulating the relevant experiment using as energy density $\varphi_{\beta}^{q c}$, instead of $\varphi$ (see [9], [10]). These will be pursued in forthcoming work. Theorems 5 and 6 provide a rigorous mathematical proof of the legitimacy of such approach in the case of the geometrically linear theory.

\section{Appendix 1: Calculus of Variations tools}

In this paper we deal with several notions of partial convexity of functions defined on matrices. For this subject our main references are [11], [19]. We denote by $\mathbb{M}^{n \times n}$ the set of $n \times n$ matrices and by $\mathbb{M}_{0}^{n \times n}$ the subset of those with zero trace. We recall that $f: \mathbb{M}^{n \times n} \mapsto \mathbb{R} \cup\{+\infty\}$ is rank-1 convex if $f\left(s \xi_{1}+(1-s) \xi_{2}\right) \leq s f\left(\xi_{1}\right)+(1-s) f\left(\xi_{2}\right)$ for every $s \in[0,1]$, $\xi_{1}, \xi_{2} \in \mathbb{M}^{3 \times 3}$ with $\operatorname{rank}\left(\xi_{1}-\xi_{2}\right) \leq 1$. A function $f: \mathbb{M}^{n \times n} \mapsto \mathbb{R} \cup\{+\infty\}$ is said to be polyconvex, if there exists a convex function $h$ such that $f(\mathbf{F})=$ $h(M(\mathbf{F}))$, where $M(\mathbf{F})$ is the vector of all the minors of $\mathbf{F}$. The definition of quasiconvexity [1] is given below.

Definition 1. A continuous function $f: \mathbb{M}^{n \times n} \mapsto \mathbb{R}$ is quasiconvex if for every $\omega$ open, bounded subset of $\mathbb{R}^{n}, \mathbf{A} \in \mathbb{M}^{n \times n}$, and $\mathbf{w} \in C_{o}^{1}\left(\omega, \mathbb{R}^{n}\right)$, we have

$$
f(\mathbf{A}) \leq|\omega|^{-1} \int_{\omega} f(\mathbf{A}+\nabla \mathbf{w}(y)) d y .
$$

We define the convex envelope of a function $f$ as $f^{c}(\xi):=\sup \{g(\xi)$ : $g \leq f, g$ convex $\}$. In the same way we define the poly-, quasi- and rank-oneconvex envelopes, by requiring that the functions $g$ in the definition above enjoy the corresponding property of partial convexity. A characterization of $f^{r c}$, which is valid also for extended-valued functions and is given in (47) below, plays a crucial role in our developments. We follow [11, Sect. 6.4] and we start with some preliminary definitions (see [11, Sect. 5.2.5]). 
Definition. For any integer $K$, let us write

$$
\Lambda_{K}:=\left\{\bar{\lambda}=\left(\lambda_{1}, \ldots, \lambda_{K}\right): \lambda_{i} \geq 0, \sum_{i}^{K} \lambda_{i}=1\right\}
$$

Consider $\bar{\lambda} \in \Lambda_{K}$ and let $\xi_{i} \in \mathbb{M}^{n \times n}, 1 \leq i \leq K$. We say that $\left\{\lambda_{i}, \xi_{i}\right\}_{i=1}^{K}$ satisfy $\left(H_{K}\right)$ if (by induction on the index $i$ )

- when $K=2$, then $\operatorname{rank}\left(\xi_{1}-\xi_{2}\right) \leq 1$;

- when $K>2$, then, up to a permutation, $\operatorname{rank}\left(\xi_{1}-\xi_{2}\right) \leq 1$ and if, for every $2 \leq i \leq K-1$, we define

$$
\left\{\begin{array}{cc}
\mu_{1}=\lambda_{1}+\lambda_{2} & \eta_{1}=\frac{\lambda_{1} \xi_{1}+\lambda_{2} \xi_{2}}{\lambda_{1}+\lambda_{2}} \\
\mu_{i}=\lambda_{i+1} & \eta_{i}=\xi_{i+1}
\end{array}\right.
$$

then $\left\{\mu_{i}, \eta_{i}\right\}_{i=1}^{K}$ satisfy $\left(H_{K-1}\right)$.

For any $f: \mathbb{M}^{n \times n} \mapsto \mathbb{R} \cup\{+\infty\}$ one can characterize $f^{r c}$ as [11, Thm 6.10]

$$
f^{r c}(\xi)=\inf \left\{\sum_{i}^{K} \lambda_{i} f\left(\xi_{i}\right): \bar{\lambda} \in \Lambda_{K}, \sum_{i}^{K} \lambda_{i} \xi_{i}=\xi,\left\{\lambda_{i}, \xi_{i}\right\} \text { satisfy }\left(H_{K}\right)\right\} .
$$

If we restrict our attention to the case of real valued functions, the following chain of inequalities follows by definition (see [11], page 265)

$$
f^{c} \leq f^{p c} \leq f^{q c} \leq f^{r c} .
$$

If $f: \mathbb{M}^{n \times n} \mapsto \mathbb{R} \cup\{+\infty\}$ the inequality $f^{q c} \leq f^{r c}$ needs not hold.

Definition 2. Let $\mathcal{F}$ be a functional defined on $H^{1}\left(\Omega, \mathbb{R}^{n}\right)$. We define the relaxation $\overline{\mathcal{F}}$ of $\mathcal{F}$ in the weak sequential (in brief, ws) topology of $H^{1}\left(\Omega, \mathbb{R}^{n}\right)$ as the largest $H^{1}\left(\Omega, \mathbb{R}^{n}\right)$-ws-lower-semicontinuous (in brief, $H^{1}\left(\Omega, \mathbb{R}^{n}\right)$-wslsc) functional below $\mathcal{F}$ :

$$
\overline{\mathcal{F}}:=\sup \left\{\mathcal{G}: \mathcal{G} \text { is } H^{1}\left(\Omega, \mathbb{R}^{n}\right) \text {-wslsc }, \mathcal{G} \leq \mathcal{F}\right\}
$$

We can now recall the main result in [7]. In what follows, for $\mathbf{v} \in$ $H^{1}\left(\Omega, \mathbb{R}^{n}\right)$ and $\Gamma$ an open subset of $\partial \Omega$ with positive $(n-1)$-surface measure, we denote by $\mathbf{v}+H_{\Gamma}^{1}\left(\Omega, \mathbb{R}^{n}\right)$ the functions in the Sobolev space $H^{1}\left(\Omega, \mathbb{R}^{n}\right)$ taking the value $\mathbf{v}$ on $\Gamma$ (in the sense of traces).

Theorem 3. Let $\Omega \subset \mathbb{R}^{n}(n=2,3)$ be an open, bounded, connected and Lipschitz set and denote with $\Gamma \subseteq \partial \Omega$ an open subset with positive ( $n-$ 
1)-surface measure. Let $f: \mathbb{M}^{n \times n} \rightarrow[0,+\infty)$ be a continuous function satisfying

$$
\begin{gathered}
c_{1}|\operatorname{sym} \mathbf{A}|^{2}-c_{2} \leq f(\mathbf{A}), \\
f(\mathbf{A}) \leq c_{3}|\operatorname{sym} \mathbf{A}|^{2}+c_{4}, \\
\left|f\left(\mathbf{A}_{1}\right)-f\left(\mathbf{A}_{2}\right)\right| \leq c_{5}\left(c_{6}+\left|\operatorname{sym} \mathbf{A}_{1}\right|+\left|\operatorname{sym} \mathbf{A}_{2}\right|\right)\left|\operatorname{sym} \mathbf{A}_{1}-\operatorname{sym} \mathbf{A}_{2}\right|,
\end{gathered}
$$

for every $\mathbf{A}, \mathbf{A}_{1}, \mathbf{A}_{2} \in \mathbb{M}^{n \times n}$, where $c_{i}=c_{i}(n), i=1, \ldots, 6$ are positive constants. Let $\kappa \in \mathbb{N}$ and define on $H^{1}\left(\Omega, \mathbb{R}^{n}\right)$

$\mathcal{F}^{\kappa}(\mathbf{u})=\int_{\Omega}\left[f(\nabla \mathbf{u})+\kappa(\operatorname{div} \mathbf{u})^{2}\right] d x, \quad \mathcal{F}(\mathbf{u})=\left\{\begin{array}{cc}\int_{\Omega} f(\nabla \mathbf{u}) d x & \text { if } \operatorname{div} \mathbf{u}=0, \\ +\infty & \text { otherwise. }\end{array}\right.$

Then, we have

$$
\overline{\mathcal{F}}=\sup _{\kappa \in \mathbb{N}} \overline{\mathcal{F}}
$$

and

$$
\overline{\mathcal{F}}(\mathbf{u})=\left\{\begin{array}{cc}
\int_{\Omega} g(\nabla \mathbf{u}) d x & \text { if } \operatorname{div} \mathbf{u}=0 \\
+\infty & \text { otherwise }
\end{array}\right.
$$

where

$$
g(\mathbf{A})=\sup _{\kappa \in \mathbb{N}}\left(\left(f(\mathbf{A})+\kappa(\operatorname{tr} \mathbf{A})^{2}\right)^{q c}\right) .
$$

Moreover, let $\mathbf{v}(x) \in H^{1}\left(\Omega, \mathbb{R}^{n}\right)$ with $\operatorname{div} \mathbf{v}=0$ a.e. in $\Omega$ and define $\mathcal{F}^{\mathbf{v}}$ by setting $\mathcal{F}^{\mathbf{v}}=\mathcal{F}$ on $\mathbf{v}+H_{\Gamma}^{1}\left(\Omega, \mathbb{R}^{n}\right)$ and $+\infty$ outside. Then the relaxation of $\mathcal{F}^{\mathbf{v}}$ is equal to $\overline{\mathcal{F}}$ on $\mathbf{v}+H_{\Gamma}^{1}\left(\Omega, \mathbb{R}^{n}\right)$ and $+\infty$ outside. Finally, g satisfies the following solenoidal quasiconvexification formula: for every $\mathbf{A} \in \mathbb{M}_{0}^{n \times n}$,

$$
g(\mathbf{A})=\inf _{\mathbf{w} \in \mathcal{A}}\left\{\frac{1}{|\omega|} \int_{\omega} g(\mathbf{A}+\nabla \mathbf{w}(y)) d y\right\}
$$

where

$$
\mathcal{A}:=\left\{\mathbf{w} \in H_{0}^{1}\left(\omega, \mathbb{R}^{n}\right): \operatorname{div} \mathbf{w}=0\right\}
$$

and $\omega$ is any open, bounded, connected and Lipschitz subset of $\mathbb{R}^{n}$. Formula (53) holds as well if we replace $H_{0}^{1}\left(\omega, \mathbb{R}^{n}\right)$ in $(54)$ by either $W_{0}^{1, \infty}\left(\omega, \mathbb{R}^{n}\right)$ or $C_{c}^{\infty}\left(\omega, \mathbb{R}^{n}\right)$.

Proof. The results above can be obtained by repeating the proof of [7, Theorem 2] in the case $n=3$. Moreover, the same proof holds for the case $n=2$ as well, since all the arguments and the technical propositions required are valid also for a domain in $\mathbb{R}^{2}$ and for two-dimensional vector fields. The possibility of choosing different spaces of amissible perturbations (54) is discussed in [7, Remark 6]. 


\section{Appendix 2: relaxation theorems}

In this section, devoted to the proof of two relaxation theorems, we use notation and tools introduced in Section 5. We always deal here with plane strain conditions (14)-(16). Hence, we do not introduce new symbols for the upper $2 \times 2$ submatrices of the matrices $\mathbf{E}_{0}(\mathbf{n})$ used in Section 3 and write

$$
\mathbf{E}_{0}\left(\mathbf{e}_{2}\right)=\frac{3}{2} \gamma\left[\begin{array}{cc}
-1 / 2 & 0 \\
0 & 1 / 2
\end{array}\right], \quad \mathbf{E}_{0}(\mathbf{n})=\frac{3}{2} \gamma\left(\mathbf{n} \otimes \mathbf{n}-\frac{1}{2} \mathbf{I}\right) .
$$

Given any matrix $\mathbf{F} \in \mathbb{M}^{2 \times 2}$ we can uniquely decompose it as

$$
\mathbf{F}=\mathbf{E}+\frac{\operatorname{tr} \mathbf{F}}{2} \mathbf{I}+\mathbf{W}
$$

where $\mathbf{W}=\operatorname{skw} \mathbf{F}=\left(\mathbf{F}-\mathbf{F}^{T}\right) / 2$, and $\mathbf{E}$ can be parametrized by

$$
\mathbf{E}:=(\operatorname{sym} \mathbf{F})_{d}=\mathbf{E}_{0}\left(\mathbf{e}_{2}\right)+\Delta \mathbf{E}=\mathbf{E}_{0}\left(\mathbf{e}_{2}\right)+\frac{3}{2} \gamma\left[\begin{array}{cc}
\varepsilon & \delta \\
\delta & -\varepsilon
\end{array}\right]
$$

with

$$
\varepsilon=\varepsilon(\mathbf{F})=\frac{1}{3 \gamma}\left(F_{11}-F_{22}\right)+\frac{1}{2}, \quad \delta=\delta(\mathbf{F})=\frac{1}{3 \gamma}\left(F_{12}+F_{21}\right) .
$$

With the aim of computing the quasiconvex envelope of $\varphi_{\beta}$, given by (20), we introduce the function $h_{\beta}: \mathbb{M}^{2 \times 2} \rightarrow[0,+\infty)$ defined as

$$
\begin{array}{r}
h_{\beta}(\mathbf{F}):=\varphi_{\beta}(\mathbf{E})+\kappa(\operatorname{tr} \mathbf{F})^{2}= \\
\inf _{\mathbf{n} \in \mathbb{S}^{1}} \mu(1+\beta)\left|\Delta \mathbf{E}+\mathbf{E}_{0}\left(\mathbf{e}_{2}\right)-\frac{1}{1+\beta} \mathbf{E}_{0}(\mathbf{n})\right|^{2}+ \\
+\frac{9}{2} \beta \gamma^{2} \mu\left[-\frac{\beta}{4(1+\beta)}\right]-2 \beta \mu \Delta \mathbf{E}: \mathbf{E}_{0}\left(\mathbf{e}_{2}\right)+\kappa(\operatorname{tr} \mathbf{F})^{2} .
\end{array}
$$

Here $\kappa$ is a positive (and finite) constant and $h_{\beta}$ is the compressible version of $\varphi_{\beta}$, trivially extended to nonsymmetric matrices. Being independent of $\operatorname{skw} \mathbf{F}, h_{\beta}$ can be written as a function of $\varepsilon, \delta, \operatorname{tr} \Delta \mathbf{F}$. Explicitly:

$$
\begin{gathered}
\widetilde{h_{\beta}}(\varepsilon, \delta, \operatorname{tr} \Delta \mathbf{F}):=\frac{9}{2} \mu \gamma^{2}\left(r(\varepsilon, \delta)-\frac{1}{2}\right)^{2}+\frac{9}{2} \beta \mu \gamma^{2}\left(\varepsilon^{2}+\delta^{2}\right)+\kappa(\operatorname{tr} \mathbf{F})^{2}= \\
\frac{9}{2} \mu \gamma^{2}(1+\beta)\left(r(\varepsilon, \delta)-\frac{1}{2} \frac{1}{1+\beta}\right)^{2}+\frac{9}{2} \mu \beta \gamma^{2}\left(\varepsilon-\frac{1}{4} \frac{\beta}{1+\beta}\right)+\kappa(\operatorname{tr} \mathbf{F})^{2},
\end{gathered}
$$

where $r(\varepsilon, \delta)$ is given by (22). Then, let us define

$$
\widetilde{j}_{\beta}(\varepsilon, \delta, \operatorname{tr} \mathbf{F}):= \begin{cases}\widetilde{h_{\beta}}(\varepsilon, \delta, \operatorname{tr} \mathbf{F}) & \text { if } r(\varepsilon, \delta) \geq \frac{1}{2} \frac{1}{1+\beta} \\ \frac{9}{2} \beta \mu \gamma^{2}\left(\varepsilon-\frac{1}{4} \frac{\beta}{1+\beta}\right)+\kappa(\operatorname{tr} \mathbf{F})^{2} & \text { else }\end{cases}
$$


and, finally,

$$
j_{\beta}(\Delta \mathbf{F}):=\widetilde{j}_{\beta}(\varepsilon(\Delta \mathbf{F}), \delta(\Delta \mathbf{F}), \operatorname{tr} \Delta \mathbf{F}) .
$$

We remark that the first summand in (60) is proportional to the distance of point $(\varepsilon, \delta)$ from the circle of center $(1 / 2,0)$ and radius $\frac{1}{2} \frac{1}{1+\beta}$. Thus, $\widetilde{h_{\beta}}$ (and hence $h_{\beta}$ ) in non-convex. On the other hand, the function

$$
(\varepsilon, \delta) \rightarrow \begin{cases}\left(r(\varepsilon, \delta)-\frac{1}{2+2 \beta}\right)^{2} & \text { if } r(\varepsilon, \delta) \geq \frac{1}{2} \frac{1}{1+\beta} \\ 0 & \text { else },\end{cases}
$$

which is easy to recognize in the definition (61) of $\widetilde{j}_{\beta}$, is precisely the square of the euclidean distance of the point $(\varepsilon, \delta)$ from the closed disk of center $(1 / 2,0)$ and radius $\frac{1}{2} \frac{1}{1+\beta}$. Since the square of the distance from a convex manifold is a convex function, it turns out that both $\tilde{j_{\beta}}$ and $j_{\beta}$ are convex. In fact, $\widetilde{j_{\beta}}$ is the convex envelope of $\widetilde{h_{\beta}}$. In turn, $j_{\beta}$ is the convex envelope of $h_{\beta}$, which coincides with the quasiconvex envelope $h_{\beta}^{q c}$. These facts are proved in the next proposition.

Proposition 4. Let $\Delta \mathbf{F} \in \mathbb{M}^{2 \times 2}, h_{\beta}$ as in (59) and $j_{\beta}$ as in (62). Then $h_{\beta}^{q c}=h_{\beta}^{c}=j_{\beta}$.

Proof. To compute the quasiconvex envelope of $h_{\beta}$ we show that

$$
\left(h_{\beta}\right)^{r c} \leq j_{\beta} \leq\left(h_{\beta}\right)^{c} \leq\left(h_{\beta}\right)^{r c},
$$

which, together with (48), implies that $\left(h_{\beta}\right)^{q c}=\left(h_{\beta}\right)^{c}=j_{\beta}$ The last inequality in (63) follows from (48). Then, a straightforward computation shows that $\widetilde{j}_{\beta} \leq \widetilde{h}_{\beta}$ and hence $j_{\beta} \leq h_{\beta}$. Therefore, the second inequality follows since $j_{\beta}$ is convex. We are only left with the proof of the first inequality in (63), which requires an explicit construction.

First of all, if $r(\varepsilon, \delta)>\frac{1}{2} \frac{1}{1+\beta}$, there is nothing to prove. Suppose then

$$
r(\varepsilon, \delta)=\sqrt{\left(\varepsilon-\frac{1}{2}\right)^{2}+\delta^{2}} \leq \frac{1}{2} \frac{1}{1+\beta} .
$$

In what follows we execute a lamination construction, in other words, we exhibit two matrices $\mathbf{F}_{1}, \mathbf{F}_{2}$ with $\operatorname{rank}\left(\mathbf{F}_{1}-\mathbf{F}_{2}\right) \leq 1$ and a real constant $\nu \in[0,1]$ such that

$$
\mathbf{F}=(1-\nu) \mathbf{F}_{1}+\nu \mathbf{F}_{2}
$$

and $j_{\beta}(\mathbf{F})=(1-\nu) h_{\beta}\left(\mathbf{F}_{1}\right)+\nu h_{\beta}\left(\mathbf{F}_{2}\right)$. To this end, let us define

$$
\Delta \mathbf{E}_{1}=\frac{3}{2} \gamma\left[\begin{array}{cc}
\varepsilon & \bar{\delta} \\
\bar{\delta} & -\varepsilon
\end{array}\right], \quad \Delta \mathbf{E}_{2}=\frac{3}{2} \gamma\left[\begin{array}{cc}
\varepsilon & -\bar{\delta} \\
-\bar{\delta} & -\varepsilon
\end{array}\right],
$$




$$
\Delta \mathbf{W}=\frac{3}{2} \gamma\left[\begin{array}{cc}
0 & \bar{\delta} \\
-\bar{\delta} & 0
\end{array}\right], \quad \widetilde{\mathbf{W}}=\mathbf{W}-(1-2 \nu) \Delta \mathbf{W}
$$

where

$$
\bar{\delta}:=\sqrt{\left(\frac{1}{2} \frac{1}{1+\beta}\right)^{2}-\left(\varepsilon-\frac{1}{2}\right)^{2}}
$$

and

$$
\nu:=\frac{1}{2}\left(1-\frac{\delta}{\bar{\delta}}\right)
$$

Notice that, in view of $(64), \nu \in[0,1]$. Moreover, $\delta=(1-\nu) \bar{\delta}+\nu(-\bar{\delta})$ and $\Delta \mathbf{E}=(1-\nu) \Delta \mathbf{E}_{1}+\nu \Delta \mathbf{E}_{2}$. Finally, let

$$
\begin{aligned}
& \mathbf{F}_{1}:=\mathbf{E}_{0}\left(\mathbf{e}_{2}\right)+\Delta \mathbf{E}_{1}+\frac{(\operatorname{tr} \mathbf{F})}{2} \mathbf{I}+\Delta \mathbf{W}+\widetilde{\mathbf{W}}, \\
& \mathbf{F}_{2}:=\mathbf{E}_{0}\left(\mathbf{e}_{2}\right)+\Delta \mathbf{E}_{2}+\frac{(\operatorname{tr} \mathbf{F})}{2} \mathbf{I}-\Delta \mathbf{W}+\widetilde{\mathbf{W}} .
\end{aligned}
$$

We observe that $\mathbf{F}=(1-\nu) \mathbf{F}_{1}+\nu \mathbf{F}_{2}$ and that $\operatorname{rank}\left(\mathbf{F}_{1}-\mathbf{F}_{2}\right) \leq 1$. A straightforward computation shows that

$$
h_{\beta}\left(\mathbf{F}_{1}\right)=h_{\beta}\left(\mathbf{F}_{2}\right)=\frac{9}{2} \mu \beta \gamma^{2}\left(\varepsilon-\frac{1}{4} \frac{\beta}{1+\beta}\right)+\kappa(\operatorname{tr} \mathbf{F})^{2}=j_{\beta}(\mathbf{F}) .
$$

Now, thanks to the representation formula (47), we have

$$
\left(h_{\beta}\right)^{r c}(\mathbf{F}) \leq(1-\nu) h_{\beta}\left(\mathbf{F}_{1}\right)+\nu h_{\beta}\left(\mathbf{F}_{2}\right)=j_{\beta}(\mathbf{F}) .
$$

This proves the claim.

\subsection{Compressible elastomers}

A direct application of Proposition 4 gives the relaxation result for the compressible case.

Theorem 5. Let $\Omega \subset \mathbb{R}^{2}$ be an open, bounded, connected and Lipschitz set and denote with $\Gamma \subseteq \partial \Omega$ an open subset with positive measure. Let $h_{\beta}, j_{\beta}$ as in (59) and (62) respectively. Let $\mathbf{v} \in H^{1}\left(\Omega, \mathbb{R}^{2}\right)$ and, for every $\mathbf{u} \in H^{1}\left(\Omega, \mathbb{R}^{2}\right)$, define

$$
\begin{gathered}
\mathrm{H}_{\beta}(\mathbf{u}):=\int_{\Omega} h_{\beta}(\nabla \mathbf{u}) d x, \quad \mathrm{~J}_{\beta}(\mathbf{u}):=\int_{\Omega} j_{\beta}(\nabla \mathbf{u}) d x, \\
\mathrm{H}_{\beta}^{\mathbf{v}}(\mathbf{u}):=\left\{\begin{array}{cc}
\mathrm{H}_{\beta}(\mathbf{u}) & \text { if } \mathbf{u} \in \mathbf{v}+H_{\Gamma}^{1}\left(\Omega, \mathbb{R}^{2}\right), \\
+\infty & \text { otherwise }
\end{array}\right.
\end{gathered}
$$




$$
\mathrm{J}_{\beta}^{\mathbf{v}}(\mathbf{u}):=\left\{\begin{array}{cc}
\mathrm{J}_{\beta}(\mathbf{u}) & \text { if } \mathbf{u} \in \mathbf{v}+H_{\Gamma}^{1}\left(\Omega, \mathbb{R}^{2}\right), \\
+\infty & \text { otherwise. }
\end{array}\right.
$$

Then, the relaxation $\overline{\mathrm{H}}_{\beta}$ and $\overline{\mathrm{H}}_{\beta}^{\mathrm{v}}$ of $\mathrm{H}_{\beta}$ and $\mathrm{H}_{\beta}^{\mathrm{v}}$ are given by

$$
\overline{\mathrm{H}}_{\beta}=\mathrm{J}_{\beta}, \quad \overline{\mathrm{H}}_{\beta}^{\mathrm{v}}=\mathrm{J}_{\beta}^{\mathrm{v}} .
$$

Moreover,

$$
\inf _{H^{1}\left(\Omega, \mathbb{R}^{2}\right)} \mathrm{H}_{\beta}^{\mathbf{v}}=\min _{H^{1}\left(\Omega, \mathbb{R}^{2}\right)} \mathrm{J}_{\beta}^{\mathbf{v}} .
$$

Proof. The results above are an application of well-known relaxation theorems (see [1] and [6, Theorem 2.3 ]). The relaxation of $\mathrm{H}_{\beta}$ (and of $\mathrm{H}_{\beta}^{v_{o}, \partial \Omega_{D}}$ ) coincides with the integral of the quasiconvex envelope of $h_{\beta}$. By Proposition 4, we have that $\left(h_{\beta}\right)^{q c}=j_{\beta}$.

\subsection{Incompressible elastomers}

Theorem 6. Let $\Omega \subset \mathbb{R}^{2}$ be an open, bounded, connected and Lipschitz set and denote with $\Gamma \subseteq \partial \Omega$ an open subset with positive measure. Let $h_{\beta}, j_{\beta}$ as in (59) and (62), respectively, and $\mathrm{H}_{\beta}, \mathrm{J}_{\beta}$ as in (72). Let $\mathbf{v} \in H^{1}\left(\Omega, \mathbb{R}^{2}\right)$ with $\operatorname{div} \mathbf{v}=0$. We define for every $\mathbf{u} \in H^{1}\left(\Omega, \mathbb{R}^{2}\right)$

$$
\begin{aligned}
& \mathcal{H}_{\beta}(\mathbf{u}):=\left\{\begin{array}{cc}
\mathrm{H}_{\beta}(\mathbf{u}) & \text { if } \operatorname{div} \mathbf{u}=0, \\
+\infty & \text { otherwise }
\end{array}\right. \\
& \mathcal{H}_{\beta}^{\mathbf{v}}(\mathbf{u}):=\left\{\begin{array}{cc}
\mathrm{H}_{\beta}^{\mathbf{v}}(\mathbf{u}) & \text { if } \operatorname{div} \mathbf{u}=0, \\
+\infty & \text { otherwise }
\end{array}\right. \\
& \mathcal{J}_{\beta}(\mathbf{u}):=\left\{\begin{array}{cc}
\mathrm{J}_{\beta}(\mathbf{u}) & \text { if } \operatorname{div} \mathbf{u}=0, \\
+\infty & \text { otherwise }
\end{array}\right. \\
& \mathcal{J}_{\beta}^{\mathbf{v}}(\mathbf{u}):=\left\{\begin{array}{cc}
\mathrm{J}_{\beta}^{\mathbf{v}}(\mathbf{u}) & \text { if } \text { div } \mathbf{u}=0, \\
+\infty & \text { otherwise }
\end{array}\right.
\end{aligned}
$$

Then, the relaxation $\overline{\mathcal{H}}_{\beta}$ and $\overline{\mathcal{H}}_{\beta}^{\mathrm{v}}$ of $\mathcal{H}_{\beta}$ and $\mathcal{H}_{\beta}^{\mathrm{v}}$ are given by

$$
\overline{\mathcal{H}}_{\beta}=\mathcal{J}_{\beta}, \quad \overline{\mathcal{H}}_{\beta}^{\mathbf{v}}=\mathcal{J}_{\beta}^{\mathbf{v}} .
$$

Moreover,

$$
\inf _{H^{1}\left(\Omega, \mathbb{R}^{2}\right)} \mathcal{H}_{\beta}^{\mathbf{v}}=\min _{H^{1}\left(\Omega, \mathbb{R}^{2}\right)} \mathcal{J}_{\beta}^{\mathbf{v}}
$$


and $j_{\beta}$ satisfies the following solenoidal quasiconvexification formula: for every $\mathbf{A} \in \mathbb{M}_{0}^{2 \times 2}$,

$$
j_{\beta}(\mathbf{A})=\inf _{\mathbf{w} \in \mathcal{A}}\left\{\frac{1}{|\omega|} \int_{\omega} h_{\beta}(\mathbf{A}+\nabla \mathbf{w}) d x\right\}
$$

where

$$
\mathcal{A}:=\left\{\mathbf{w} \in C_{c}^{\infty}\left(\omega, \mathbb{R}^{2}\right): \operatorname{div} \mathbf{w}=0\right\}
$$

and $\omega \subset \mathbb{R}^{2}$ is any open, bounded, connected and Lipschitz set. Formula (83) holds as well if we replace $H_{0}^{1}\left(\omega, \mathbb{R}^{n}\right)$ in (84) by either $W_{0}^{1, \infty}\left(\omega, \mathbb{R}^{n}\right)$ or $C_{c}^{\infty}\left(\omega, \mathbb{R}^{n}\right)$.

Proof. It is sufficient to apply Theorem 3 with $n=2$. It is easy to check that $h_{\beta}$ satisfies $(50-51)$. Moreover, recalling that the euclidean distance is a Lipschitz function, (52) follows.

\subsection{Summary}

We summarize here some of the results of this Section, and recast them in the notation of Section 3. Setting, for any $\mathbf{A} \in \mathbb{M}^{2 \times 2}$,

$$
\varphi_{\beta}(\mathbf{A})=\left\{\begin{array}{cc}
h_{\beta}(\mathbf{A}) & \text { if } \operatorname{tr} \mathbf{A}=0 \\
+\infty & \text { if } \operatorname{tr} \mathbf{A} \neq 0
\end{array}\right.
$$

and

$$
\left(\varphi_{\beta}\right)^{q c}(\mathbf{A})=\left\{\begin{array}{cc}
j_{\beta}(\mathbf{A}) & \text { if } \operatorname{tr} \mathbf{A}=0, \\
+\infty & \text { if } \operatorname{tr} \mathbf{A} \neq 0
\end{array}\right.
$$

we recover formulas (25)-(28).

We have also a formula for the quasiconvex envelope in the compressible case. Setting, for any $\mathbf{A} \in \mathbb{M}^{2 \times 2}$,

$\tilde{\varphi}_{\beta}(\mathbf{A})=\mu\left|(\operatorname{sym} \mathbf{A})_{d}-\mathbf{E}_{0}(\mathbf{n})\right|^{2}+\beta \mu\left|(\operatorname{sym} \mathbf{A})_{d}-\mathbf{E}_{0}\left(\mathbf{e}_{2}\right)\right|^{2}+\frac{\kappa}{2}(\operatorname{tr} \mathbf{A})^{2}=h_{\beta}(\mathbf{A})$

we have

$$
\left(\tilde{\varphi}_{\beta}\right)^{q c}(\mathbf{A})=\left(\varphi_{\beta}\right)^{q c}\left(\mathbf{A}_{d}\right)+\frac{\kappa}{2}(\operatorname{tr} \mathbf{A})^{2}=j_{\beta}(\mathbf{A}) .
$$

The reader is referred to [22] for results on compressible elastomers within the fully nonlinear theory.

Acknowledgments. ADS thanks Mariarita de Luca and Luciano Teresi for valuable discussions and help during the preparation of this manuscript. 


\section{References}

[1] E. Acerbi, N. Fusco, 1984. Semicontinuity problems in the calculus of variations, Arch. Rat. Mech. Anal. 86, 125-145.

[2] V. Agostiniani, A. DeSimone: Gamma-convergence of energies for nematic elastomers in the small strain limit, forthcoming (2010).

[3] K. Bhattacharya. Microstructure of Martensite. Oxford University Press, Oxford, 2003.

[4] J.S. Biggins, E.M. Terentjev, M. Warner, 2008. Semisoft elastic response of nematic elastomers to complex deformations, Phys. Rev. E 78, 041704.1-9.

[5] Bladon, P., Terentjev, E. M., Warner, M., 1993. Transitions and instabilities in liquid-crystal elastomers. Phys. Rev. E 47, R3838-R3840.

[6] Braides, A., 1987. Relaxation of functionals with constraints on the divergence. Ann. Univ. Ferrara - Sez. VII - Sc. Mat. XXXIII 157-177.

[7] Cesana, P., 2010. Relaxation of multi-well energies in linearized elasticity and applications to nematic elastomers. Arch. Rat. Mech. Anal. 197, 903-923.

[8] Cesana, P., DeSimone, A., 2009. Strain-order coupling in nematic elastomers: equilibrium configurations. Math. Models Methods Appl. Sci. 19, 601-630.

[9] Conti, S., DeSimone, A., Dolzmann, G., 2002. Soft elastic response of stretched sheets of nematic elastomers: a numerical study. J. Mech. Phys. Solids 50, 1431-1451.

[10] Conti, S., DeSimone, A., Dolzmann, G., 2002. Semi-soft elasticity and director reorientation in stretched sheets of nematic elastomers. Phys. Rev. E 60, 61710-1-8.

[11] B. Dacorogna, Direct methods in the Calculus of Variations 2ed, Springer, 2008.

[12] DeSimone, A., 1999. Energetics of fine domain structures. Ferroelectrics $222,275-284$.

[13] DeSimone, A., 2010. Electro-Mechanical Response of Nematic Elastomers: an Introduction. CISM Lecture Notes, Springer Verlag, forthcoming. 
[14] A. DeSimone, G. Dolzmann, 2002. Macroscopic response of nematic elastomers via relaxation of a class of $\mathrm{SO}(3)$-invariant energies. Arch. Rat. Mech. Anal. 161, 181-204.

[15] A. DeSimone, L. Teresi, 2009. Elastic energies for nematic elastomers. Eur. Phys. J. E 29, 191-204.

[16] Finkelmann, H., Kundler, I., Terentjev, E.M., Warner, M., 1997. Critical stripe-domain instability of nematic elastomers, J. Phys. II France 7, 1059-1069.

[17] A. Fukunaga, K. Urayama, T. Takigawa, A. DeSimone, L. Teresi, 2008. Dynamics of Electro-Opto-Mechanical Effects in Swollen Nematic Elastomers. Macromolecules 41, 9389-9396.

[18] Kundler, I., Finkelmann, H., 1995. Strain-induced director reorientation in nematic liquid single crystal elastomers. Macromol. Rapid Comm. 16, 679-686.

[19] S. Müller, 1999. Variational methods for microstructure and phase transitions, in: Proc. C.I.M.E. summer school "Calculus of variation and geometric evolution problems", Cetraro 1996, (F. Bethuel, G. Huisken, S. Müller, K. Steffen, S. Hildebrandt, M. Strüwe eds.), Springer LNM vol. 1713 .

[20] A. Petelin, M. Copic, 2009. Observation of a Soft Mode of Elastic Instability in Liquid Crystal Elastomers. Phys. Rev. Lett. 103, 077801-1-4.

[21] D. Rogez, G. Francius, H. Finkelmann, P. Martinoty, 2006. Shear mechanical anisotropy of side chain liquid-crystal elastomers: Influence of sample preparation. Eur. Phys. J. E 20, 369-378.

[22] Silhavy, M., 2007. Ideally soft nematic elastomers. Networks and Heterogeneous Media 2, 279-311.

[23] Verwey, M. Warner, E. Terentjev, 1996. Elastic instability and stripe domains in liquid crystalline elastomers. J. Phys. II France 6, 12731290.

[24] Warner, M., Terentjev, E. M. Liquid Crystal Elastomers. Revised Edition, Clarendon Press, Oxford, 2007. 